Pacific Journal of Mathematics

ORDER-PRESERVING FUNCTIONS: APPLICATIONS TO

ALBeRT W. MARShalL, David WiLLIam WaLk U 


\title{
ORDER-PRESERVING FUNCTIONS; APPLICATIONS TO MAJORIZATION AND ORDER STATISTICS
}

\begin{abstract}
A. W. Marshall, D. W. Walkup and R. J.-B. Wets
Let $\lesssim$ be a partial ordering among the points of a set $D \subset R^{n}$. A real-valued function $f$ defined on $D$ is said to preserve $\lesssim$ if $x, y \in D, x \precsim y$ implies $f(x) \leqq f(y)$. The central theorem of this paper gives necessary and sufficient conditions for $f$ to preserve $\precsim$ if $\precsim$ is a cone ordering, i.e. if there exists a convex cone $C$ such that $x \precsim y$ if and only if $y-x \in C$. Corollaries to the theorem consider the case when $f$ is differentiable and $\lesssim$ is order isomorphic to a cone ordering under a differentiable mapping. It is seen that the ordering of majorization is a special case of a cone ordering and that a straightforward application of a corollary yields the results of Schur and Ostrowski on functions which preserve majorization. The corollaries are also applied to a partial ordering of positive semi-definite matrices and to certain partial orderings arising in the theory of order statistics.
\end{abstract}

In order to prove and extend the determinant inequality of Hadamard, I. Schur [22] defined a relation that is equivalent to the ordering of majorization [10, p. 49]. A real $n$-vector $y$ is said to majorize the $n$-vector $x$ (written $x \prec y$ ) if the components of $x$ and $y$ can be reordered so that

(i ) $x_{1} \geqq x_{2} \geqq \cdots \geqq x_{n}, y_{1} \geqq y_{2} \geqq \cdots \geqq y_{n}$,

(ii) $\sum_{i=1}^{k} x_{i} \leqq \sum_{i=1}^{k} y_{i}, \quad k=1,2, \cdots, n-1$,

(iii) $\sum_{i=1}^{n} x_{i}=\sum_{i=1}^{n} y_{i}$.

Schur showed that a real-valued differentiable function $f$ preserves the ordering $\prec$, i.e. $x \prec y$ implies $f(x) \leqq f(y)$, if and only if $f$ is symmetric and

(iv) $\frac{\partial f(x)}{\partial x_{i}}-\frac{\partial f(x)}{\partial x_{i+1}} \geqq 0, \quad i=1,2, \cdots, n-1$,

for all $x$ satisfying (i). ${ }^{1}$ With the choice of particular functions $f$ and the insight that the eigenvalues of a positive definite Hermitian matrix majorize its diagonal elements, he was able to obtain the determinant inequality of Hadamard as well as a number of related inequalities.

The importance of Schur's approach to the Hadamard inequality lies in the fact that his results and their refinements due to A. Ostrowski

${ }^{1}$ Actually, Schur restricted his attention to the case that $x_{i}>0, y_{i}>0$; this restriction was removed by Ostrowski [19]. 
[19] can be applied to obtain a variety of inequalities wherever majorization arises. In matrix theory majorization also occurs in the comparison of eigenvalues with singular values [24], [11] and in the study of incidence matrices [21]. Majorization has also found applications in other contexts, e.g. in obtaining certain probabilistic inequalities [18], [20]. Other partial orderings occur in the study of condition numbers for matrices [16], order statistics [17], and positive definite matrices [14]. In each case the problem arises of determining necessary and sufficient conditions for a real-valued function $f$ to be order preserving.

The purpose of this paper is to show how such conditions can be derived in general. Our approach is illustrated by the following simple interpretation of the ordering of majorization and Schur's condition. Vectors $x$ and $y$ satisfy (ii) and (iii) if and only if $y-x$ belongs to the convex cone $C$ contained in the hyperplane $\sum_{i=1}^{n} z_{i}=0$ and determined by the inequalities $\sum_{i=1}^{k} z_{i} \geqq 0, k=1, \cdots, n-1$. Then (iv), as we shall see, is equivalent to the requirement that at each point $x$ satisfying (i), the directional derivative of $f(x)$ is nonnegative in the direction of the edges of the convex cone $C$.

Section 2 is devoted to obtaining general conditions for a realvalued function to be order-preserving when the partial ordering is a cone ordering or is order isomorphic to a cone ordering. Section 3, by way of illustration, applies those results to the ordering of majorization and partial orderings found in [3], [14], and [17].

2. Theory. A relation $\lesssim$ on a set $D$ is a partial ordering if

$$
\begin{aligned}
& x \precsim x \text { for all } x \text { in } D \quad \text { (reflexitivity) } \\
& x \precsim y, y \precsim z \text { implies } x \precsim z \quad \text { (transitivity) . }
\end{aligned}
$$

Note that the condition

$$
x \precsim y, y \precsim x \text { implies } x=y \quad \text { (antisymmetry) }
$$

is not required.

A convex cone is a nonempty set $C \subset R^{n}$ such that $p, q \in C$ implies $\lambda_{1} p+\lambda_{2} q \in C$ for all $\lambda_{1}, \lambda_{2} \geqq 0$. A convex cone $C$ is said to be pointed if $x \in C,-x \in C$ implies $x=0$. A cone ordering on $D \subset R^{n}$ (induced by a convex cone $C$ ) is a relation $\precsim$ on $D$ defined by:

$$
x \precsim y \text { if and only if } y-x \in C \text {. }
$$

Any cone ordering $\precsim$ on $R^{n}$ is a partial ordering and satisfies

$$
x \precsim y \text { implies } x+z \precsim y+z \text {, for all } x, y, z \text { in } R^{n}
$$

$$
x \precsim y \text { implies } \lambda x \precsim \lambda y \text {, for all } x, y \text { in } R^{n} \text { and } \lambda \geqq 0 \text {. }
$$


Conversely, if $\precsim$ is a partial ordering on $R^{n}$ satisfying (3) and (4), then $\precsim$ is the cone ordering induced by the convex cone $C=\{x: x \succsim 0\}$. Moreover, $C$ is pointed if and only if $\precsim$ satisfies (1).

A set $S \subset C$ is said to span the convex cone $C$ positively if every point of $C$ can be expressed as a positive linear combination of a finite number of points in $S$. A set $T \subset C$ is called a frame of $C$, if $T$, but no proper subset of $T$, spans $C$ positively. A convex cone which possesses a finite frame is called a convex polyhedral cone.

It can be shown (though we shall not specifically require this result) that every closed convex cone $C$ in $R^{n}$ possesses a frame, but not necessarily a finite one. In particular, any frame for $C$ can be obtained by combining a (necessarily finite) frame for the maximal linear subspace $L$ contained in $C$ (see McKinney [15]) and a frame for the pointed cone $C \bmod L$. The existence of a frame for a pointed closed convex cone in $R^{n}$ follows from an application of the KreinMilman theorem to a bounded set obtained by intersecting the cone with an appropriate linear variety. Results along this line have been obtained by Klee [13].

A particularly simple but important cone ordering is the componentwise ordering $\leqq$ on $R^{n}$ given by

$$
x \leqq y \text { if and only if } x_{i} \leqq y_{i} \text { for } i=1, \cdots, n,
$$

where $R^{n}$ is interpreted as the space of column $n$-tuples and $x_{i}$ denotes the $i^{\text {th }}$ component of $x$. It is readily checked that $\leqq$ is the cone ordering induced by the positive orthant $R_{+}^{n}=\{x: x \geqq 0\}$, and that the unit coordinate vectors $e^{i}, i=1, \cdots, n$, constitute a frame for $R_{+}^{n}$.

In applications the cone $C$ is often given in terms of $m$ simultaneous linear inequalities, i.e. $C=\{x: A x \geqq 0\}$ where $A$ is an $m \times n$ matrix, According to a theorem of Weyl [8], [9], $C$ is a convex polyhedral cone. In general, the task of computing a frame for $C$ given the $m \times n$ matrix $A$ is not easy; it is equivalent to determining the extreme points of a convex polytope given the equations of bounding hyperplanes, or vice versa. See for example [2] and [23]. However, the matter is simple when $A$ is nonsingular.

Proposition 1. If the matrix $A$ is nonsingular, the columns of $A^{-1}$ constitute a frame for the convex cone $C=\{x: A x \geqq 0\}$.

Proof. The cone $C=\{x: A x=y, y \geqq 0\}$ can be rewritten as

$$
C=\left\{x: x=A^{-1} y, y \geqq 0\right\} \text {. }
$$

Thus the columns of $A^{-1}$ span $C$ positively. But clearly no proper subset spans $C$. 
Our fundamental result on cone orderings is contained in the following

THEOREM 2. Suppose $f$ is a real-valued function defined on a convex set $D \subset R^{n}$ with interior, $f$ is continuous at boundary points of $D$ and $\precsim$ is a cone ordering on $D$ induced by the convex cone $C \subset R^{n}$ with frame $T$. Then $f$ preserves $\precsim$ if and only if

$$
\begin{aligned}
& f(x+\lambda t)-f(x) \geqq 0, \text { for all } x \in D \\
& \text { and all } t \in T \text { and } \lambda>0 \text { such that } x+\lambda t \in D .
\end{aligned}
$$

Proof. "Only if" is obvious. Suppose therefore that (5) holds. Fix $x, y \in D$ such that $x \precsim y$. We must show $f(y)-f(x) \geqq 0$. Since $v=y-x \in C$, there are $t_{1}, \cdots, t_{k}$ in $T$ and positive scalars $\mu_{1}, \cdots, \mu_{k}$ such that

$$
v=\sum_{i=1}^{k} \mu_{i} t_{i}
$$

If $x, y \in \operatorname{int} D$ (the interior of $D$ ), then for suitable $\alpha>0$ the cylindrical neighborhood (See Figure 1.)

$$
E=\left\{z \in R^{n}:\|z-w\|<\alpha \text { for some } w \text { on the line segment }[x, y]\right\}
$$

is contained in $D$, where $\|\cdot\|$ is the Euclidean norm on $R^{n}$. See Figure 1. It is now easy to see that there exists an integer $N$ and a polyhedral path from $x$ to $y$ lying entirely in $E$ and having $N k+1$ nodes, each of which is displaced from the preceding node by one of the vectors $(1 / N) \mu_{i} t_{i}, i=1, \cdots, k$.

By (5), $f$ is nondecreasing along each arc of the path, hence $f(y)-f(x) \geqq 0$. Next suppose $x$ and $y$ are points of $D$ on the boundary. Let $z \in \operatorname{int} D$, and for each $\delta \in(0,1)$ define

$$
x_{\delta}=(1-\delta) x+\delta z, y_{\delta}=(1-\delta) y+\delta z \text {. }
$$

Since $f$ is continuous at $x$ and $y$, for each $\varepsilon>0$ there exists some $\delta$ such that $f\left(x_{\delta}\right) \geqq f(x)-\varepsilon$ and $f\left(y_{\delta}\right) \leqq f(y)+\varepsilon$. Moreover, $x_{\delta} \precsim y_{\delta}$ with $x_{\delta}, y_{\delta} \in \operatorname{int} D$. Hence by the argument above, $f\left(y_{\delta}\right)-f\left(x_{\delta}\right) \geqq 0$. It follows that $f(y)-f(x) \geqq-2 \varepsilon$ for all $\varepsilon>0$, thus $f(y)-f(x) \geqq 0$. If exactly one of the points $x, y$ lies in int $D$, essentially the same proof applies if $z$ is taken to be the one in the interior.

The following theorem shows that the hypotheses on $D$ in Theorem 2 are essentially the weakest that can be imposed without involving $C$.

THEOREM 3. Suppose $D$ is a nonempty closed subset of $R^{n}$ such that, for every convex cone $C$ with frame $T$ and every continuous 

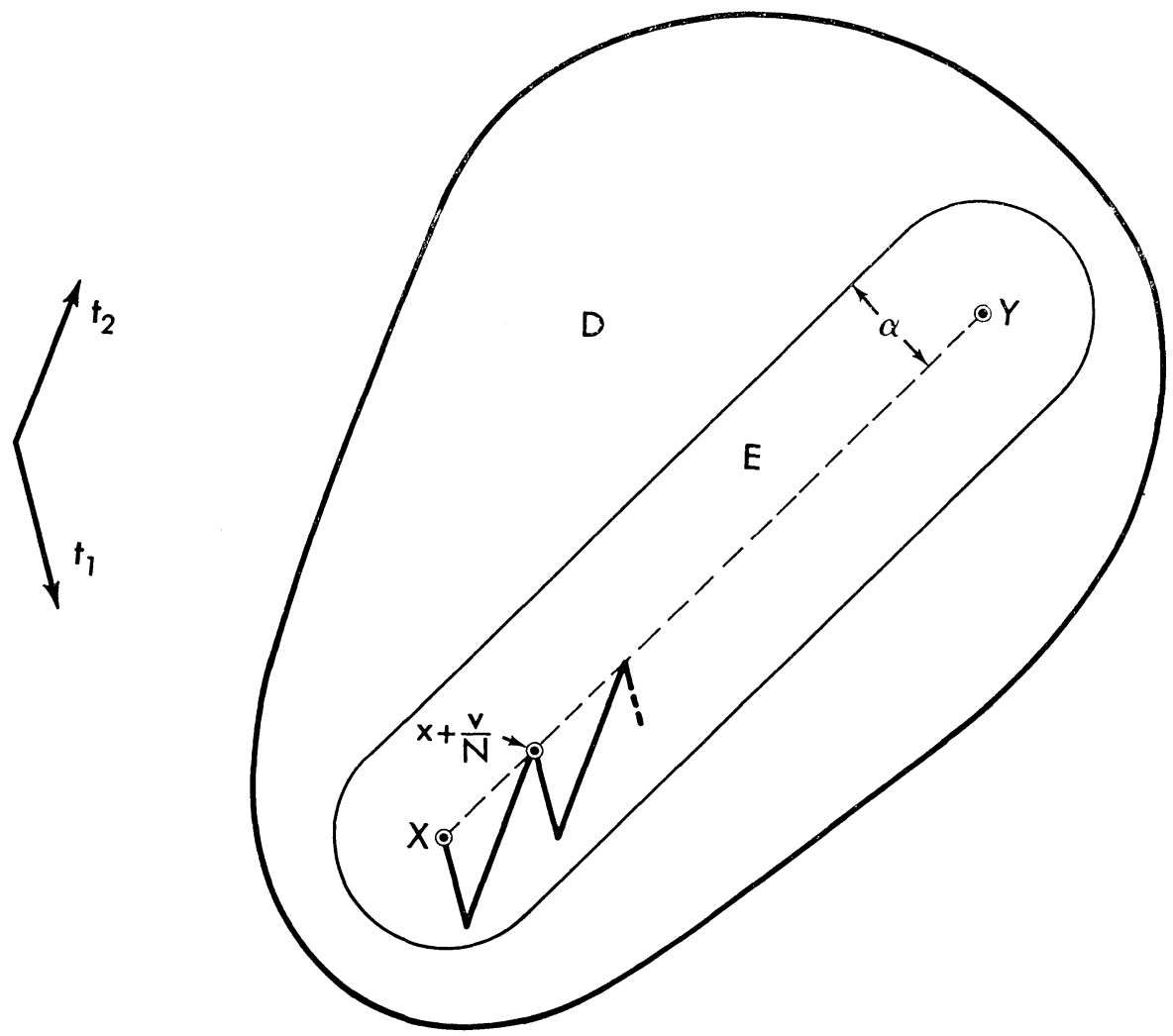

FIGURE 1.

function $f$ on $D$, condition (5) is sufficient to insure that $f$ preserves the cone ordering on $D$ induced by $C$. Then $D$ is a point or $D$ is convex with nonempty interior.

Outline of proof. It suffices to show that a "counterexample" to Theorem 2 can be constructed whenever $D$ has at least two points and is not convex with interior. We illustrate the construction for $n=2$ when $D$ is not convex; the generalization to dimension $n$ involves no essential difficulties. Consider the closed subset $D$ of $R^{2}$ shown shaded in Figure 2. Since $D$ is not convex there exist points $p$ and $q$ on the boundary of $D$ such that the open interval $(p, q)$ is contained in the complement of $D$ (which is open). Select $p^{\prime}, q^{\prime}$ sufficiently close to $p$ and $q$ respectively on the extension of the line segment $[p, q]$, and vectors $t_{1}, t_{2}$ sufficiently close to parallel to $q-p$. Let $C$ be the convex cone spanned by $\left\{t_{1}, t_{2}\right\}$ and let $\precsim$ be the cone ordering on $R^{2}$ induced by $C$. If $p^{\prime}, q^{\prime}, t_{1}$, and $t_{2}$ are properly chosen, there exist distinct points $p^{\prime \prime}, q^{\prime \prime}$ in $[p, q]$ such that $p^{\prime} \precsim p^{\prime \prime} \precsim q^{\prime \prime} \precsim q^{\prime}$ and such that $x \in D$ and $p^{\prime} \precsim x \precsim q^{\prime}$ implies $p^{\prime} \precsim x \precsim p^{\prime \prime}$ or $q^{\prime \prime} \precsim x \precsim q^{\prime}$, i.e. the open set $E$ in Figure 2 is disjoint from $D$. A continuous function $f$ 
can be defined on the complement of $E$ as follows: $f$ is constant on the unstriped regions in Figure 2, with value 0 or 1 as indicated, and linearly interpolated on the striped regions. It can be verified that the restriction of $f$ to $D$ satisfies (5), but $f$ is not order preserving since $p \precsim q$ and $f(p)>f(q)$.

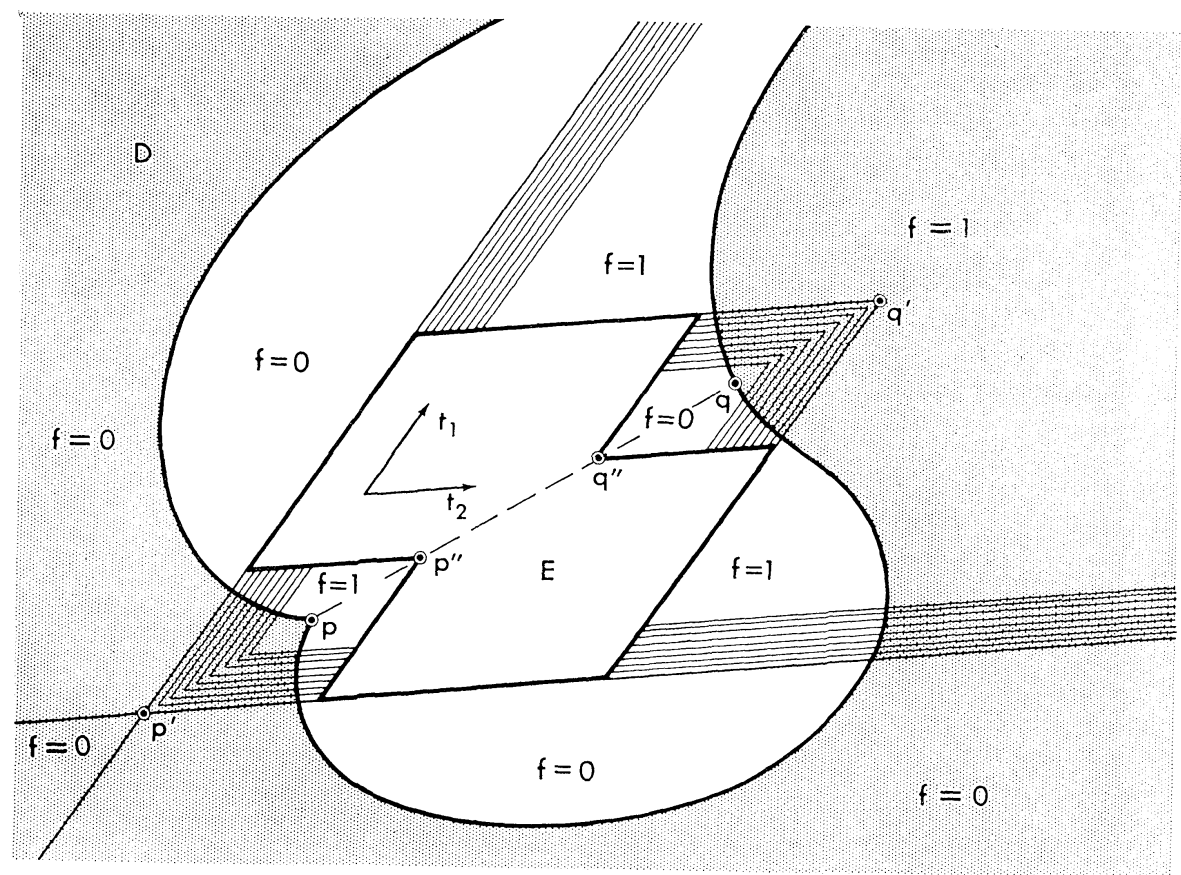

FIGURE 2.

It remains to be observed that if $D$ is a convex set without interior, then any function $f$ will satisfy (5) trivially if the convex cone $C$ and its frame $T$ are chosen so that no member of $T$ is parallel to a line segment in $D$.

In application, it is generally more convenient to use a differential form of Theorem 2. For any function $f$ defined on $D \subset R^{n}$, we adopt the convention that the gradient

$$
\nabla f(x)=\left(\frac{\partial f(x)}{\partial x_{1}}, \cdots, \frac{\partial f(x)}{\partial x_{n}}\right)
$$

at $x \in \operatorname{int} D$ is a row vector, since it is a point of the dual of the column vector space $R^{n}$. This convention will make transposition of vectors unnecessary when writing inner products, as in equation (6) below, where $\nabla f(x) \cdot t$ denotes the inner product of $\nabla f(x)$ and $t$.

CoROLlary 4. Suppose $f$ is a continuous real-valued function defined on a convex set $D \subset R^{n}$ with gradient $\nabla f(x)$ at each point in 
the nonempty interior of $D$, and $\lesssim$ is a cone ordering on $D$ induced by the convex cone $C \subset R^{n}$ with frame $T$. Then $f$ preserves $\precsim$ if and only if

$$
\nabla f(x) \cdot t \geqq 0
$$

for all $t$ in $T$ and all $x$ in the interior of $D .^{2}$

Proof. It is clear from the proof that Theorem 2 remains true when condition (5) is modified to read "...for all $x \in$ int $D \ldots$... To say that $\nabla f(x)$ exists at $x \in \operatorname{int} D$ is to say that the directional derivative $\lim _{\lambda \downarrow 0}[f(x+\lambda t)-f(x)] / \lambda$ can be expressed as the inner product $\nabla f(x) \cdot t$ (in fact, the convergence is uniform in $t$ for $t$ in a bounded neighborhood of the origin). The corollary now follows from the relationship between the directional derivative and monotonicity of $f$ on line segments $[x, x+\lambda t], t \in T, x+\lambda t \in D$.

We remark that Corollary 4 remains true if, in the last line, $T$ is replaced by a dense subset of $T$ or a dense subset of a frame $T^{\prime}$ obtained from $T$ by normalization. A similar remark is valid for Theorem 2 provided $f$ is required to be continuous.

It may happen that a partial ordering $\precsim$ on a set $D \subset R^{n}$ is not a cone ordering, but can be related to one. Specifically, suppose $G$ is a one-to-one mapping of $D$ onto $G(D) \subset R^{n}$ and that $\nwarrow_{C}$ is a cone ordering on $G(D)$ such that $x \precsim y$ if and only if $G(x) \preccurlyeq_{C} G(y)$. Such a $G$ can be viewed as an order isomorphism between $(D, \precsim)$ and $\left(G(D), \precsim_{C}\right)$. It is clear that a function $f$ defined on $D$ preserves $\precsim$ if and only if the composite function $\phi=f G^{-1}$ preserves $\lessgtr_{C}$. Since $G$ maps into $R^{n}$, it can be described in terms of its $n$ real-valued coordinate functions $g_{i}$, where $g_{i}(x)$ is the $i^{\text {th }}$ coordinate of $G(x)$. We give some conditions on these $g_{i}$ sufficient to insure that Corollary 4 and the chain rule of differentiation may be applied to $f G^{-1}$.

Definition 5. Let $\mathscr{G}_{n}$ be the class of all mappings $G$ such that

(i) the domain $D \subset R^{n}$ of $G$ is contained in the closure of its interior,

(ii) $G$ is a homeomorphism of $D$ onto $G(D) \subset R^{n}$,

(iii) on int $D, G$ is of class $C^{1}$ and the derivative matrix

$$
J_{G}(x)=\left(\frac{\partial g_{i}(x)}{\partial x_{j}}\right)
$$

is nonsingular.

${ }^{2}$ Although, in the interest of simplicity, we avoid introducing the concept of the polar (dual) $C^{*}$ of a convex cone $C$, we cannot help remarking that equation (6) is equivalent to the statement that $\nabla f(x)$ belongs to $C^{*}=\{z: z \cdot y \geqq 0$, for all $y \in C\}$. 
Proposition 6. If $G \in \mathscr{G}_{n}$, then $G(\operatorname{int} D)=\operatorname{int} G(D), G^{-1} \in \mathscr{G}_{n}$, and $J_{G^{-1}}(G(x))=\left[J_{G}(x)\right]^{-1}$ for all $x \in \operatorname{int} D$.

Proof. From the Invariance of Domain Theorem [12, p. 95; or 1, p. 156] it follows that $G(\operatorname{int} D)=\operatorname{int} G(D)$. The rest of the proposition follows from the simpler results for the restrictions of $G$ and $G^{-1}$ to the open sets int $D$ and int $G(D)$ respectively. See for example [6, Sections 5.5, 5.7].

It is now obvious from Corollary 4 and Proposition 6 that:

Corollary 7. Suppose $G \in \mathscr{G}_{n}$ has domain $D$ and convex range $G(D), \Im_{C}$ is a cone ordering on $G(D)$ induced by the convex cone $C$ with frame $T$, and $\lesssim$ is a partial ordering on $D$ such that $x \lessgtr y$ if and only if $G(x) \preccurlyeq_{C} G(y)$. If $f$ is a continuous real-valued function defined on $D$ with gradient $\nabla f$ on the interior of $D$, then $f$ preserves $\precsim$ if and only if

$$
\nabla f(x) \cdot\left[J_{G}(x)\right]^{-1} \cdot t \geqq 0
$$

for all $t$ in $T$ and all $x$ in the interior of $D$.

In Corollary 7, $G$ may be interpreted as a reparametrization of $D$ which converts $\lesssim$ into a cone ordering. A somewhat different interpretation is possible when the coordinate functions $g_{i}$ are used to define the partial ordering $\lesssim$ on $D$ as in

CoROLlaRY 8. Suppose $\precsim$ is a partial ordering on a set $D \subset R^{n}$ given by

$$
x \lesssim y \text { if and only if: } \begin{aligned}
& g_{i}(x) \leqq g_{i}(y), 1 \leqq i \leqq k \\
& g_{i}(x)=g_{i}(y), k<i \leqq n-l .
\end{aligned}
$$

Suppose further that the mapping $G$ whose coordinate functions are $g_{1}, \cdots, g_{n}$ belongs to $\mathscr{G}_{n}$ and has convex range $G(D) \subset R^{n}$. If $f$ is a continuous real-valued function defined on $D$ with gradient $\nabla f$ on the interior of $D$, then $f$ preserves $\precsim$ if and only if the first $k$ components of the vector $\nabla f \cdot\left[J_{G}(x)\right]^{-1}$ are nonnegative and the last $l$ components are zero for all $x$ in the interior of $D$.

Proof. Corollary 7 applies with

$$
C=\left\{z: z_{i} \geqq 0,1 \leqq i \leqq k, \text { and } z_{i}=0, k<i \leqq n-l\right\} .
$$

Thus it suffices to observe that the vectors 


$$
\begin{gathered}
e^{i}, 1 \leqq i \leqq k, \\
e^{i}, n-l<i \leqq n, \\
-e^{i}, n-l<i \leqq n,
\end{gathered}
$$

constitute a frame for the cone $C$.

3. Applications. We apply the results of $\S 2$ to four examples of vector partial orderings, together with certain minor variations. The first example is the ordering of majorization already investigated by Schur [22], Ostrowski [19], and others. The second two examples arise in the theory of order statistics and here some of the results are new. The fourth example is the matrix ordering induced by the cone of positive semi-definite matrices, which has been studied by Loewner [14].

In all cases we confine ourselves to the characterization of order preserving functions which are continuous on a set $D$ and have a gradient on the interior of $D$. This must be understood, although to avoid repetitions we do not continue to mention it.

EXAMPLE 1. In the notation of $\S 2$, let

$$
D=\left\{x \in R^{n}: x_{1} \geqq x_{2} \geqq \cdots \geqq x_{n}\right\} .
$$

On $D$, define

Order 1a. $x \lessgtr_{1 a} y$ if and only if

$$
\sum_{i=1}^{k} x_{i} \leqq \sum_{i=1}^{k} y_{i}, k=1,2, \cdots, n, x, y \in D .
$$

This is a cone ordering since $x \lessgtr_{1 a} y$ if and only if $y-x \in C=$ $\{z: A z \geqq 0\}$ where $A=\left[a_{i j}\right]$ is an $n \times n$ lower triangular matrix with $a_{i j}=1$ for $i \geqq j$ and $a_{i j}=0$ for $i<j$. By Proposition 1 and Corollary 4, $f$ preserves $\lesssim_{1 a}$ if and only if $\nabla f(x) A^{-1} \geqq 0$ for $x \in \operatorname{int} D$. Since $A^{-1}=\left[\alpha_{i j}\right]$, where $\alpha_{i j}=1$ if $i=j, \alpha_{i j}=-1$ if $i=j+1$ and $\alpha_{i j}=0$ otherwise, we have

Condition 1a. $f$ preserves $\lessgtr_{1 a}$ if and only if

$$
\frac{\partial f(x)}{\partial x_{1}} \geqq \frac{\partial f(x)}{\partial x_{2}} \geqq \cdots \geqq \frac{\partial f(x)}{\partial x_{n}} \geqq 0
$$

for all $x$ in the interior of $D$.

In the literature more attention has been devoted to a modification of $\lessgtr_{1 a}$, defined on the same set $D$, namely: 
Order 1b. $x \Im_{1 b} y$ if and only if

$$
\sum_{i=1}^{k} x_{i} \leqq \sum_{i=1}^{k} y_{i}, k=1,2, \cdots, n-1, \text { and } \sum_{i=1}^{n} x_{i}=\sum_{i=1}^{n} y_{i}, x, y \in D \text {. }
$$

We apply Corollary 8 with $k=n-1, l=0$, and $G(x)=A x$ where $A$ is the same as for order 1a. Since $\left[J_{G}(x)\right]^{-1}=A^{-1}$ it follows that

Condition 1b. $f$ preserves $\precsim_{1 b}$ if and only if

$$
\frac{\partial f(x)}{\partial x_{1}} \geqq \frac{\partial f(x)}{\partial x_{2}} \geqq \cdots \geqq \frac{\partial f(x)}{\partial x_{n}}
$$

for all $x$ in the interior of $D$.

Any partial ordering $\precsim$ on $D=\left\{x: x_{1} \geqq x_{2} \geqq \cdots \geqq x_{n}\right\}$ can be extended to a partial ordering $\Im^{\dagger}$ on $R^{n}$ by defining $x \prec^{\dagger} y$ if $x^{\dagger} \prec y^{\dagger}$, where $x^{\dagger}$ is the vector obtained from $x$ by rearranging the components in decreasing order. We note the obvious result:

PRoposition 9. If $\precsim$ is a partial ordering on

$$
D=\left\{x: x_{1} \geqq x_{2} \geqq \cdots \geqq x_{n}\right\}
$$

then a function $f$ preserves $\nwarrow^{\dagger}$ if and only if $f$ is symmetric and preserves $\precsim$.

Thus, $f$ preserves $\lessgtr_{1 a}^{\dagger}$ (or $\lessgtr_{1 b}^{\dagger}$ ) if $f$ is symmetric and satisfies condition $1 a$ (or condition $1 b$ ). This result was obtained by Ostrowski [19]. The partial ordering $\nwarrow_{1 a}^{\dagger}$ has also been considered by K. Fan [7] who showed that $\phi(x) \leqq \phi(y)$ for all symmetric gauge functions $\phi$ if and only if $x \lesssim_{1 a}^{\dagger} y$. The ordering $\lesssim_{1 b}^{\dagger}$ (i.e., majorization) has been discussed by Hardy, Littlewood and Pólya [10, pp. 44-49, p. 89], and by Beckenbach and Bellman [4, pp. 30-32]. The reader of [4] should note that $\S 29$, p. 31 applies to the partial ordering $\S_{1 b}^{\dagger}$ and $\S 31$, p. 32 applies to $\precsim_{1 b}$.

EXAMPLE 2. Let $D_{+}=\left\{x \in R^{n}: x_{1} \geqq x_{2} \geqq \cdots \geqq x_{n}>0\right\}$. We define

Order $2 a . \quad x \S_{2 a} y$ if and only if

$$
\frac{x_{i}}{x_{i+1}} \leqq \frac{y_{i}}{y_{i+1}}, i=1, \cdots, n-1, x, y \in D_{+} .
$$

A geometric interpretation of $\Im_{2 a}$ can be given. If $x, y \in \operatorname{int} D_{+}$, then $x \precsim_{2 a} y$ if and only if there exists a starshaped function $\phi$ such that $y_{i}=\phi\left(x_{i}\right)$. A function $\phi$ defined on $(0, \infty)$ is starshaped if the line segment joining a point on the graph of $\phi$ to the origin lies entirely 
on or above the graph.

The partial ordering $\lessgtr_{2 a}$ has arisen in the theory of reliability [17]. In [5] a justification is given for saying that a component "wears out" if the distribution $F$ of its time to failure is starshaped with respect to the exponential distribution $H$, i.e. if $H^{-1} F$ is starshaped. Order statistics $X_{1}>X_{2}>\cdots>X_{n}>0$ and $Y_{1}>Y_{2}>\cdots>Y_{n}>0$ from such distributions $F$ and $H$, respectively, have the property that $X_{i} / X_{i+1}$ is stochastically less then $Y_{i} / Y_{i+1}$. The functions preserving $\S_{2 a}$ are of particular interest, since they are exactly those yielding tests of the hypothesis of no wear-out which are unbiased at all levels of significance. Moreover, it has been shown in [3] that $\left(E X_{1}, E X_{2}, \cdots, E X_{n}\right) \lessgtr_{2 a}\left(E Y_{1}, E Y_{2}, \cdots, E Y_{n}\right)$.

In order to apply Corollary 8 to $\precsim_{2 a}$, we take $g_{i}(x)=x_{i} / x_{i+1}$ for $i=1, \cdots, n-1$. There is some latitude in choosing $g_{n}$. A convenient choice is $g_{n}(x)=x_{n}$. The map $G$ whose coordinate functions are $g_{1}, \cdots, g_{n}$ takes $D_{+}$homeomorphically onto the convex set

$$
\left\{z: z_{i} \geqq 1, i=1, \cdots, n-1, z_{n}>0\right\},
$$

which has interior. The inverse of $G$ is given by $x_{k}=\prod_{i=k}^{n} g_{i}(x)$. It is readily computed that $\left[J_{G}(x)\right]^{-1}=J_{\sigma^{-1}}(G(x))=\left[a_{i j}\right]$, where $a_{i j}=$ $x_{i} x_{j+1} / x_{j}$ for $1 \leqq i \leqq j<n, a_{i j}=0$ for $1 \leqq j<i \leqq n$, and $a_{i_{n}}=x_{i} / x_{n}$ for $1 \leqq i \leqq n$. From Corollary 8 with $k=n-1$, and $l=1$ we have

Condition 2a. $f$ preserves $\lessgtr_{2 a}$ if and only if

$$
\begin{aligned}
& \sum_{i=1}^{j} x_{i} \frac{\partial f(x)}{\partial x_{i}} \geqq 0, \quad j=1, \cdots, n-1 \\
& \sum_{i=1}^{n} x_{i} \frac{\partial f(x)}{\partial x_{i}}=0
\end{aligned}
$$

for all $x \in \operatorname{int} D_{+}$.

Note that since $g_{1}, \cdots, g_{n-1}$ are homogeneous, any function $f$ which preserves $\S_{2 a}$ must satisfy $f(x)=f(\lambda x)$ for all $\lambda>0$, or equivalently $[\partial f(\lambda x) / \partial \lambda]_{\lambda=1}=0$, which is just (10).

If we define

Order $2 b . \quad x \nwarrow_{2 b} y$ if and only if

$$
x \Im_{2 a} y \text { and } x_{n} \leqq y_{n}, x, y \in D_{+},
$$

then by a minor variation of the application of Corollary 8 to $\lessgtr_{2 a}$, we have

Condition 2b. $f$ preserves $\lessgtr_{2 b}$ if and only if 


$$
\sum_{i=1}^{j} x_{i} \frac{\partial f(x)}{\partial x_{i}} \geqq 0, \quad j=1, \cdots, n
$$

for all $x \in \operatorname{int} D_{+}$.

This result can be obtained by an alternate method. Write $u_{i}=\ln x_{i}$ and $v_{i}=\ln y_{i}$ so that $u$ and $v$ range over

$$
D=\left\{u: u_{1} \geqq u_{2} \geqq \cdots \geqq u_{n}\right\} \text {. }
$$

Then $x \preccurlyeq_{2 b} y$ if and only if $u \lessgtr_{1 c} v$, where

$$
\begin{aligned}
& u \lesssim_{1 c} v \text { if and only if } \\
& u_{i}-u_{i+1} \leqq v_{i}-v_{i+1}, i=1, \cdots, n-1 \text {, and } u_{n} \leqq v_{n}, u, v \in D .
\end{aligned}
$$

This is the cone ordering induced by the cone $C^{*}=\left\{x:\left(A^{-1}\right)^{T} x \geqq 0\right\}$, where $A$ is the matrix used in defining the cone $C$ which induces $\lesssim_{1 a}$. Hence by Proposition 1 and Corollary $4, f$ preserves $\lesssim_{1 c}$ if and only if $\nabla f(u) \cdot A^{T} \geqq 0$, i.e.

$$
\sum_{i=1}^{j} \frac{\partial f(u)}{\partial u_{i}} \geqq 0, \quad j=1, \cdots, n,
$$

for all $u \in \operatorname{int} D$. The change of variables yields Condition $2 b$. Observe that the members of the frame of $C$ (the columns of $A^{-1}$ ) determine the bounding inequalities for $C^{*}$, and conversely, the members of the frame of $C^{*}$ (the rows of $A$ ) determine the bounding inequalities for $C$. These facts reflect a duality between $\lesssim_{1 a}$ and $\precsim_{1 c}$. A similar, though not so obvious, duality exists between $\precsim_{1 b}$ (majorization) and $\lessgtr_{2 a}$ (the ordering used in [17]) expressed as a cone ordering on the $u^{\prime} s$.

In [17] the following variant of $\lesssim_{2 a}$ is also considered.

Order $2 c . \quad x \lesssim_{2 c} y$ if and only if

$$
\frac{x_{i}}{x_{i+1}} \leqq \frac{y_{i}}{y_{i+1}}, i=1, \cdots, n-1 \text {, and } \sum_{i=1}^{n} x_{i}=\sum_{i=1}^{n} y_{i}, x, y \in D_{+} \text {. }
$$

The map $G$ whose coordinate functions are

$$
g_{i}(x)=x_{i} / x_{i+1}, i=1, \cdots, n-1, g_{n}(x)=\sum_{1}^{n} x_{i}
$$

is again a homeomorphism of $D_{+}$onto the same convex set as in Example 2a. With somewhat more labor than before we find

$$
\left[J_{G}(x)\right]^{-1}=\left[a_{i j} / \sum_{1}^{n} x_{k}\right]
$$

where $a_{i j}=x_{i} x_{j+1}\left(\sum_{j+1}^{n} x_{k}\right) / x_{j}$ for $1 \leqq i \leqq j<n, a_{i j}=-x_{i} x_{j+1}\left(\sum_{1}^{j} x_{k}\right) / x_{j}$ 
for $1 \leqq j<i \leqq n$, and $a_{i n}=x_{i}$ for $1 \leqq i \leqq n$.

From Corollary 8 with $k=n-1$ and $l=0$ we have

Condition $2 c . \quad f$ preserves $\lessgtr_{2 c}$ if and only if

$$
\left(\sum_{i=1}^{j} x_{i} \frac{\partial f(x)}{\partial x_{i}}\right)\left(\sum_{k=j+1}^{n} x_{k}\right) \geqq\left(\sum_{i=j+1}^{n} x_{i} \frac{\partial f(x)}{\partial x_{i}}\right)\left(\sum_{k=1}^{j} x_{k}\right) j=1, \cdots, n-1
$$

for all $x \in \operatorname{int} D_{+}$.

We remark that the use of $g_{n}(x)=\sum_{1}^{n} x_{i}$ in computing Condition $2 a$ would have led to the more involved set of conditions (11) and (10). However, it is possible to reduce (11) to (9) with the use of (10).

Suppose two partial orderings $\nwarrow_{a}$ and $\lessgtr_{b}$ of the type considered in this paper are defined on a set $D$, and $x \preccurlyeq_{a} y$ implies $x \preccurlyeq_{b} y$. Suppose further that comparatively simple conditions are known for a function to preserve $\Im_{b}$. Then any function which satisfies these conditions preserves $\lessgtr_{a}$. This observation, coupled with the result given in Proposition 10 below, was used in [17, Th. 2.4] to show that a function satisfying (8) preserves $\nwarrow_{2 c}$. We give here an alternate proof of Proposition 10 which illustrates an interesting application of Corollary 8.

Proposition 10. If $x, y \in D_{+}$and $x \preccurlyeq_{2 c} y$, then $y$ majorizes $x$, i.e. $x \preccurlyeq_{1 b}^{\dagger} y$.

Proof. Since $D_{+}$is contained in the domain of definition of $\precsim_{1 b}$, it suffices to show that $\nwarrow_{2 c}$ is preserved by each of the functions

$$
\begin{aligned}
f_{k}(x) & =\sum_{i=1}^{k} x_{i} \quad k=1, \cdots, n-1 \\
f_{n}(x) & =\sum_{i=1}^{n} x_{i} \\
f_{n+1}(x) & =-\sum_{i=1}^{n} x_{i},
\end{aligned}
$$

used in defining $\precsim_{1 b}$. It is easily verified that these functions satisfy (11). In fact, $f_{n}$ and $f_{n+1}$ satisfy (11) with equality. This is a direct consequence of the fact $f_{n}(x)=f_{n}(y)$ is required for both $x \precsim{ }_{2 c} y$ and $x \precsim_{1 b} y$.

ExAmple 3. Let $D_{+}^{\circ}=\left\{x: x_{1}>x_{2}>\cdots>x_{n}>0\right\}$ and define

Order 3. $x \nwarrow_{3} y$ if and only if $x, y \in D_{+}^{\circ}$ and 


$$
\frac{x_{i}-x_{i+1}}{x_{i+1}-x_{i+2}} \leqq \frac{y_{i}-y_{i+1}}{y_{i+1}-y_{i+2}}, i=1, \cdots, n-2,
$$

and

$$
\frac{x_{n-1}-x_{n}}{x_{n}} \leqq \frac{y_{n-1}-y_{n}}{y_{n}}
$$

Just as $\lessgtr_{2 a}$ arises from the study of distribution functions $F$ which are starshaped with respect to a distribution $H, \precsim_{3}$ arises from distributions $F$ which are convex with respect to a distribution $H$, i.e. for which $H^{-1} F$ is convex. Observe that $x \precsim_{3} y$ if and only if $y_{i}=\phi\left(x_{i}\right)$ for some nondecreasing convex function $\phi$ such that $\phi(0)=0$. Thus $x \nwarrow_{3} y$ implies $x \S_{2 a} y$. We define the map $G$ by

$$
g_{i}(x)=\frac{x_{i}-x_{i+1}}{x_{i+1}-x_{i+2}}, i=1,2, \cdots, n,
$$

where we adopt the convention that $x_{n+1}=0$ and $x_{n+2}=-1$. G maps $D_{+}^{\circ}$ homeomorphically onto $\left\{x: x_{i}>0, i=1, \cdots, n\right\}$. We have $x_{i}=$ $\sum_{k=i}^{n} \prod_{j=k}^{n} g_{j}(x)$ and $\left[J_{G}(x)\right]^{-1}=\left[a_{i j}\right]$, where

$$
a_{i j}=\left(x_{i}-x_{j+1}\right)\left(x_{j+1}-x_{j+2}\right) /\left(x_{j}-x_{j+1}\right)
$$

for $j \geqq i$ and $a_{i j}=0$ for $j<i$. By Corollary 8 we have

Condition 3. $f$ preserves $\precsim_{3}$ if and only if

$$
\begin{gathered}
\sum_{i=1}^{j}\left(x_{i}-x_{j+1}\right) \frac{\partial f(x)}{\partial x_{i}} \geqq 0, j=1, \cdots, n-1, \\
\sum_{i=1}^{n} x_{i} \frac{\partial f(x)}{\partial x_{i}}=0
\end{gathered}
$$

for all $x$ in $D_{+}^{\circ}$.

EXAMPle 4. We identify $D=R^{n(n+1) / 2}$ with the set of all $n \times n$ symmetric matrices, and on $D$ define

Order 4. $A \lessgtr_{4} B$ if and only if $B-A$ is positive semi-definite.

This ordering, which was introduced by C. Loewner [14], is the cone ordering induced by the convex cone of positive semi-definite matrices. Though this cone does not have a finite frame, the set of all positive semi-definite matrices $M=\left[m_{i j}\right]$ of rank one, normalized by the condition trace $M=1$, does constitute a frame. Such matrices can be written in the form $m m^{T}=\left[m_{i} m_{j}\right]$ where $m$ is a unit column vector, and any positive semi-definite matrix $A$ has the representation $A=$ $\sum_{i=1}^{r} \alpha_{i} M_{i}$ where $r$ is the rank of $A$ and $\alpha_{i}>0$. 
We apply Corollary 4. Note that (6) can be written as

$$
\sum_{i, j} \frac{\partial f(A)}{\partial a_{i j}} m_{i} m_{j}=m^{T}\left[\frac{\partial f(A)}{\partial a_{i j}}\right] m \geqq 0 .
$$

Thus we obtain that

Condition 4. $f$ preserves $\preccurlyeq_{4}$ if and only if the gradient matrix $\left[\partial f(A) / \partial a_{i j}\right]$ is positive semi-definite.

A related result has been obtained by Loewner [14], who considered the case that $f$ is orthogonally invariant, i.e., $f(A)=f\left(\Gamma A \Gamma^{T}\right)$ for all orthogonal matrices $\Gamma$. He showed that such functions preserve $\lessgtr_{4}$ if and only if

$$
\frac{\partial f(A)}{\partial \lambda_{i}} \geqq 0, i=1,2, \cdots, n
$$

where $\lambda_{i}$ are the eigenvalues of $A$.

\section{REFERENCES}

1. P. S. Aleksandrov, Combinatorial Topology, Graylock Press, Rochester, New York, 1956.

2. M. Balinski, An algorithm for finding all vertices of convex polyhedral sets, J. Soc. Indust. Appl. Math. 9 (1961), 72-88.

3. R. Barlow and F. Proschan, Inequalities for linear combinations of order statistics from restricted families, Ann. Math. Statist. 37 (1966), 1574-1592.

4. E. F. Beckenbach and R. Bellman, Inequalities, Springer-Verlag, Berlin, 1961.

5. Z. W. Birnbaum, J. D. Esary and A. W. Marshall, A stochastic characterization of wear-out for components and systems, Ann. Math. Statist. 37 (1966), 816-825.

6. R. C. Buck, Advanced Calculus, McGraw-Hill, New York, 1956.

7. K. Fan, Maximum properties and inequalities for the eigenvalues of completely continuous operators, Proc. Nat. Acad. Sci. U.S.A. 37 (1951), 760-766.

8. D. Gale, Convex polyhedral cones and linear inequalities, Activity Analysis of Production and Allocation, T. C. Koopmans, ed., John Wiley, New York, 1951, 287-297. 9. M. Gerstenhaber, Theory of convex polyhedral cones, Activity Analysis of Production and Allocation, T. C. Koopmans, ed., John Wiley, New York, 1951, 298-316.

10. G. Hardy, J. Littlewood and G. Pólya, Inequalities, second edition, Cambridge University press, Cambridge, 1952.

11. A. Horn, On the singular values of a product of completely continuous operators, Proc. Nat. Acad. Sci. U.S.A. 36 (1950), 374-375.

12. W. Hurewicz and H. Wallman, Dimension Theory, Princeton University Press, Princeton, 1948.

13. V. Klee, Extremal structure of convex sets, Archiv der Mathematik 8 (1957), 234-240.

14. C. Loewner, Advanced Matrix Theory, Mimeographed notes, Stanford University, 1957.

15. R. McKinney, Positive bases for linear spaces, Trans. Amer. Math. Soc. 103 (1962), $131-148$. 
16. A. W. Marshall and I. Olkin, Norms and inequalities for condition numbers, Pacific J. Math. 15 (1965), 241-247.

17. A. W. Marshall, I. Olkin and F. Proschan, Monotonicity of ratios of means and other applications of majorization, Inequalities: Proceeding of a Sympsium, O. Shisha, ed., Academic Press, New York, 1967, 177-190.

18. A. W. Marshall and F. Proschan, An inequality for conve: functions involving majorization, J. Math. Anal. Appl. 12 (1965), 87-90.

19. A. Ostrowski, Sur quelques applications des functions convexes et concaves au sens de I. Schur, J. Math. Pure Appl. 31 (1952), 253-292.

20. F. Proschan, Peakedness of distributions of convex combinations, Ann. Math. Statist. 36 (1965), 1703-1706.

21. H. J. Ryser,Matrices of zero and ones, Bull. Amer. Math. Soc. 66 (1960), 442-464.

22. I. Schur, Über eine Klasse von Mittelbildungen mit Anwendungen auf die Determinantentheorie, Sitzber. Berl. Math. Ges. 22 (1923), 9-20.

23. R. Wets and C. Witzgall, Towards an algebraic Characterization of Convex Polyhedral Cones, Boeing document D1-82-0525, Boeing Scientific Research Laboratories, Seattle, Washington, April, 1966.

24. H. Weyl, Inequalities between the two kinds of eigenvalues of linear transformation, Proc. Nat. Acad. Sci. U.S.A. 35 (1949), 408-411.

Received January 9, 1967.

Boeing SCIEntific Research Laboratories 


\section{PACIFIC JOURNAL OF MATHEMATICS}

\section{H. ROYDEN}

Stanford University

Stanford, California

\author{
J. P. JANS \\ University of Washington \\ Seattle, Washington 98105
}

\section{EDITORS}

\author{
J. DugundJI \\ Department of Mathematics \\ Rice University \\ Houston, Texas 77001 \\ Richard ARENS \\ University of California \\ Los Angeles, California 90024
}

\section{ASSOCIATE EDITORS}
E. F. BECKENBACH
B. H. NeumanN
F. WOLF
K. YOSIDA

\section{SUPPORTING INSTITUTIONS}

\author{
UNIVERSITY OF BRITISH COLUMBIA \\ CALIFORNIA INSTITUTE OF TECHNOLOGY \\ UNIVERSITY OF CALIFORNIA \\ MONTANA STATE UNIVERSITY \\ UNIVERSITY OF NEVADA \\ NEW MEXICO STATE UNIVERSITY \\ OREGON STATE UNIVERSITY \\ UNIVERSITY OF OREGON \\ OSAKA UNIVERSITY \\ UNIVERSITY OF SOUTHERN CALIFORNIA
}

\author{
STANFORD UNIVERSITY \\ UNIVERSITY OF TOKYO \\ UNIVERSITY OF UTAH \\ WASHINGTON STATE UNIVERSITY \\ UNIVERSITY OF WASHINGTON \\ AMERICAN MATHEMATICAL SOCIETY \\ CHEVRON RESEARCH CORPORATION \\ TRW SYSTEMS \\ NAVAL ORDNANCE TEST STATION
}

Mathematical papers intended for publication in the Pacific Journal of Mathematics should be typewritten (double spaced). The first paragraph or two must be capable of being used separately as a synopsis of the entire paper. It should not contain references to the bibliography. Manuscripts may be sent to any one of the four editors. All other communications to the editors should be addressed to the managing editor, Richard Arens at the University of California, Los Angeles, California 90024.

50 reprints per author of each article are furnished free of charge; additional copies may be obtained at cost in multiples of 50 .

The Pacific Journal of Mathematics is published monthly. Effective with Volume 16 the price per volume (3 numbers) is $\$ 8.00$; single issues, $\$ 3.00$. Special price for current issues to individual faculty members of supporting institutions and to individual members of the American Mathematical Society: $\$ 4.00$ per volume; single issues $\$ 1.50$. Back numbers are available.

Subscriptions, orders for back numbers, and changes of address should be sent to Pacific Journal of Mathematics, 103 Highland Boulevard, Berkeley 8, California.

Printed at Kokusai Bunken Insatsusha (International Academic Printing Co., Ltd.), 7-17, Fujimi 2-chome, Chiyoda-ku, Tokyo, Japan.

PUBLISHED BY PACIFIC JOURNAL OF MATHEMATICS, A NON-PROFIT CORPORATION

The Supporting Institutions listed above contribute to the cost of publication of this Journal, but they are not owners or publishers and have no responsibility for its content or policies. 


\section{Pacific Journal of Mathematics}

A. A. Aucoin, Diophantine systems ............................. 419

Charles Ballantine, Products of positive definite matrices. I ............... 427

David Wilmot Barnette, A necessary condition for d-polyhedrality ............ 435

James Clark Beidleman and Tae Kun Seo, Generalized Frattini subgroups of finite groups ......................................... 441

Carlos Jorge Do Rego Borges, A study of multivalued functions ............. 451

William Edwin Clark, Algebras of global dimension one with a finite ideal

lattice ...............................................

Richard Brian Darst, On a theorem of Nikodym with applications to weak convergence and von Neumann algebras .........................

George Wesley Day, Superatomic Boolean algebras .....................

Lawrence Fearnley, Characterization of the continuous images of all

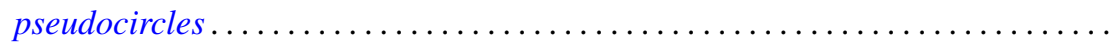

Neil Robert Gray, Unstable points in the hyperspace of connected subsets....... 515

Franklin Haimo, Polynomials in central endomorphisms .................. 521

John Sollion Hsia, Integral equivalence of vectors over local modular lattices . . . . 527

Jim Humphreys, Existence of Levi factors in certain algebraic groups .......... 543

E. Christopher Lance, Automorphisms of postliminal $C^{*}$-algebras ............ 547

Sibe Mardesic, Images of ordered compacta are locally peripherally metric . . . . 557

Albert W. Marshall, David William Walkup and Roger Jean-Baptiste Robert Wets,

Order-preserving functions: Applications to majorization and order

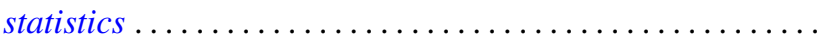

Wellington Ham Ow, An extremal length criterion for the parabolicity of

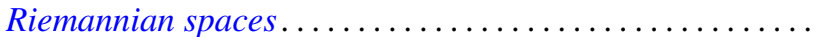

585

Wellington Ham Ow, Criteria for zero capacity of ideal boundary components of

Riemannian spaces...................................... 591

J. H. Reed, Inverse limits of indecomposable continua .................. 597

Joseph Gail Stampfli, Minimal range theorems for operators with thin spectra . . . 601

Roy Westwick, Transformations on tensor spaces..................... 613

Howard Henry Wicke, The regular open continuous images of complete metric

spaces ........................................... 621

Abraham Zaks, A note on semi-primary hereditary rings .............. 627

Thomas William Hungerford, Correction to: "A description of $\operatorname{Mult}_{i}\left(A^{1}, \cdots, A^{n}\right)$

by generators and relations" ............................. 629

Uppuluri V. Ramamohana Rao, Correction to: "On a stronger version of Wallis' formula".............................................. 629

Takesi Isiwata, Correction: "Mappings and spaces" ................... 630

Henry B. Mann, Josephine Mitchell and Lowell Schoenfeld, Correction to:

"Properties of differential forms in $n$ real variables" .... . .

James Calvert, Correction to: "An integral inequality with applications to the

Dirichlet problem"............................. 